\title{
EFFECTS OF COULOMB INTERACTIONS BETWEEN FERMIONS ON SUPERCONDUCTIVITY IN BOSON-FERMION MODEL
}

\author{
T. KOSTYRKo \\ Institute of Physics, A. Mickiewicz University \\ Umultowska 85, 61-614 Poznań, Poland
}

\begin{abstract}
Influence of Coulomb repulsion between fermions on stability of the superconducting state in the model of boson-like local electron pairs hybridized with wide-band fermions is studied. It is shown rigorously that the Bose condensation of the local pairs implies a divergence of the pairing susceptibility in the fermion subsystem. Charge carrier concentration dependence of the critical temperature $T_{\mathrm{c}}$ is evaluated with the help of random phase approximation and spectral properties of the bosons at $T_{c}$ are analyzed.
\end{abstract}

PACS numbers: 74.20.-z, 05.30.-d, 67.40.Db

The high values of $T_{\mathrm{c}}$ of superconducting $\mathrm{Cu}-\mathrm{O}$ ceramics (with relatively small concentration of charge carriers, $n$ ) being proportional to the Fermi temperature [1] and their small values of the coherence length $\xi$ are consistent with a short-range almost unretarded attraction as a possible source of electron pairing. For the pair radius smaller than the interpair distance, a condition which may hold for strong enough local attraction, the pairs (possibly formed well above $T_{\mathrm{c}}$ ) become similar to hard core charged bosons and the resulting superconducting state would resemble a Bose condensate. Indeed, the critical behavior of thermodynamic quantities in the $\mathrm{Cu}-\mathrm{O}$ superconductors supports the picture of Bose condensation $[2,3]$. On the other hand, the verified existence of the Fermi surface together with several typically "electronic" properties" of these materials (Pauli-like magnetic susceptibility and the oxygen NMR relaxation rate above $T_{c}$, antiferromagnetism in some range of $n$ ) are naturally explained within the Fermi liquid theory.

The two seemingly contradictory types of behavior may be reconciled within the model describing a system of heavy (hard core) bosons hybridized with wide-band electrons. Such a model was introduced by Ranninger and Robaszkiewicz [4] within a small polaron theory and subsequently applied by these authors $[5,2,6]$ and others $[7,8]$ as a possible explanation of properties of nonconventional superconductors.

In its most simple form $[7,6]$ the boson-fermion (BF) model may be written as 
$\mathcal{H}_{\mathrm{BF}}=\sum_{\boldsymbol{k} \sigma} \varepsilon_{\boldsymbol{k}} c_{\boldsymbol{k}_{\sigma}}^{\dagger} c_{\boldsymbol{k} \sigma}+\sum_{\boldsymbol{k}} \xi_{\boldsymbol{k}} b_{k}^{\dagger}{ }_{k} \boldsymbol{k}_{k}+\frac{v}{\sqrt{N}} \sum_{k, p}\left(b_{k} c_{k / 2+\boldsymbol{p} \uparrow}^{\dagger} c_{k / 2-\boldsymbol{p} \downarrow}^{\dagger}+\right.$ h.c. $)$

where $c_{k_{\sigma}}^{\dagger}, c_{k_{\sigma}}\left(b_{k}^{\dagger}, b_{k}\right)$ represent the fermion (boson) operators, $\varepsilon_{k}=\varepsilon_{k}^{0}-\mu, \xi_{k}=$ $\xi_{k}^{0}+\Delta_{\mathrm{B}}-2 \mu, \varepsilon_{k}^{0}\left(\xi_{k}^{0}\right)$ is a fermion (boson) band energy, $\Delta_{\mathrm{B}}-$ the bottom of the boson band, $\mu$ - the chemical potential and $N$ - the number of lattice sites (here $\hbar=k_{\mathrm{B}}=1$ ). The last sum in (1) describes a process of decay of a boson particle into a pair of fermions and the opposite process, $v$ denotes the BF coupling. In what follows the boson hard-core effects will be neglected, which is justified as long as the boson concentration is small $\left(n_{\mathrm{B}}=\frac{1}{N} \sum_{k}\left\langle b_{k}^{\dagger} b{ }_{k}\right\rangle \ll 1\right)$. The Hamiltonian is analysed subject to the total charge carrier conservation condition: $n=n_{\mathrm{F}}+2 n_{\mathrm{B}}$.

The model (1) exhibits two different types of limiting behavior depending on the concentration $n$ and the position of the boson level (it is assumed that the boson band stays above the bottom of the fermion band) [5,7]. For the reliably small interaction $v$ and small enough $n\left(n \ll n_{\mathrm{c}}\right)$ the bottom of the boson band lies above the Fermi energy and only very few of boson states can be occupied. The processes of virtual excitations of the fermions to the boson level create the effective attraction between the fermions, $g_{\text {eff }} \sim 2 v^{2} /\left(\Delta_{\mathrm{B}}-2 \mu\right)$. In this "BCS" range of $n$ the superconducting properties of the model (1) resemble very much the ones of the weak-coupling BCS superconductor. For higher $n\left(n>n_{\mathrm{c}}\right)$ the Fermi level touches the bottom of the boson band $\left(\Delta_{\mathrm{B}}=2 \mu\right)$ and all further supply of the electrons into the system goes to the boson states. In such "Bose" limit the superconducting transition is governed by the condensation of the bosons with $T_{\mathrm{c}} \sim\left(n-n_{\mathrm{c}}\right)^{\frac{2}{3}}[7]$.

The inherent property of the model (1) is that the formation of the (s-wave) condensate of fermion Cooper pairs is always accompanied by the condensation of bosons and vice versa. This can be shown by considering the equation of motion for a Green function for bosons, $G\left(q, t-t^{\prime}\right)=-\mathrm{i} \theta\left(t-t^{\prime}\right)\left\langle\left[b_{q}(t), b_{q}^{\dagger}\left(t^{\prime}\right)\right]\right\rangle \equiv$ $\left\langle\left\langle b_{q}(t) \mid b_{q}^{\dagger}\left(t^{\prime}\right)\right\rangle\right\rangle$. Differentiating $G\left(q, t-t^{\prime}\right)$ with respect to $t$ and a resulting next order Green function with respect to $t^{\prime}$ leads to an exact relation between the time-Fourier transform of $G\left(q, t-t^{\prime}\right)$ and the fermion pair susceptibility, $\chi_{\mathrm{SS}}(q, \omega)=$ $\frac{1}{N} \sum_{k, p}\left\langle\left\langle c_{-p \uparrow}^{\dagger} c_{p+q \downarrow}^{\dagger} \mid c_{k+q \downarrow} c_{-k \uparrow}\right\rangle\right\rangle_{\omega}$ :

$$
G(q, \omega)=G_{0}(q, \omega)+v^{2} G_{0}^{2}(q, \omega) \chi_{\mathrm{SS}}(q, \omega), \quad \text { where } \quad G_{0}(q, \omega) \equiv \frac{1}{\omega-\xi_{k}} .
$$

The direct consequence of Eq. (2) is the common temperature of divergence of the boson Green function and the $s$-wave pair susceptibility at $\omega, q=0$, which shows (by virtue of the Thouless criterion) that the onset of macroscopic occupation of the $q=0$ boson state is accompanied by a superconducting transition in the fermion subsystem [8].

The deficiency of the presented so far analysis of the BF model is neglect of the Coulomb repulsion which is known to be important in the cuprate superconductors and crucial for explanation of antiferromagnetic and insulating properties of their parent $\mathrm{La}_{2} \mathrm{CuO}_{4}$ and $\mathrm{YBa}_{2} \mathrm{Cu}_{3} \mathrm{O}_{6}$ compounds. It is natural to ask if $s$-wave state generated by the $\mathrm{BF}$ coupling in the form given by (1) survives the destroying 
influence of the on-site Coulomb correlations. To study this problem we complete the model (1) with a Hubbard term describing on-site repulsion between fermions*

$$
\mathcal{H}_{U}=\frac{U}{N} \sum_{k p q} c_{k+q \uparrow}^{\dagger} c_{k \uparrow} c_{p-q \downarrow}^{\dagger} c_{p \downarrow} .
$$

Including $\mathcal{H}_{U}$ into the Hamiltonian does not change Eq. (2), because $\mathcal{H}_{U}$ does not depend on boson operators. Provided that the Hubbard $U$ is not too strong as compared to a bandwidth $2 D$ one can use random phase approximation (RPA) for the quantitative estimation of $G(q, \omega)$. The RPA result for the normal phase is

$$
G^{-1}(q, \omega)=G_{0}^{-1}(q, \omega)-\Sigma_{\mathrm{B}}(q, \omega), \quad \Sigma_{\mathrm{B}}(q, \omega)=\frac{v^{2} \chi_{\mathrm{SS}}^{0}(q, \omega)}{1-U \chi_{\mathrm{SS}}^{0}(q, \omega)},
$$

where $\chi_{\mathrm{SS}}^{0}(q, \omega)=\frac{1}{N} \sum_{k}\left(n^{\mathrm{F}}{ }_{k}+n_{k_{k+q}}^{\mathrm{F}}-1\right) /\left(\bar{\varepsilon}_{k}+\bar{\varepsilon}_{k+q}-\omega\right)$ is the 0 -order pair susceptibility, $\bar{\varepsilon}_{1} k=\varepsilon_{k}+\frac{U}{2} n_{\mathrm{F}}$ and $n^{\mathrm{F}}{ }_{k}=1 /\left[\exp \left(\bar{\varepsilon}_{k} / T\right)+1\right]$. From Eq. (4) one gets the equation determining a temperature of divergence of $G(q, \omega)$ for $q, \omega=0$ :

$$
1=-\left(\frac{v^{2}}{\xi_{0}}-U\right) \chi_{\mathrm{SS}}^{0}(0,0), \quad \text { where } \quad \chi_{\mathrm{SS}}^{0}(0,0)=-\frac{1}{2 N} \sum_{k} \frac{\tanh \left(\bar{\varepsilon}_{k} / 2 T\right)}{\bar{\varepsilon}_{k}}
$$

To examine the superconducting state of the model one can apply a mean-field approximation (MFA) [7] to find a gap parameter $x$. The MFA gap equation takes a form of (5) with $\chi_{\mathrm{SS}}^{0}(0,0)$ replaced by: $-\frac{1}{2 N} \sum_{k} \tanh \left(\sqrt{\bar{\varepsilon}_{k}^{2}+x^{2}} / 2 T\right) / \sqrt{\bar{\varepsilon}_{k}^{2}+x^{2}}$ and reduces to (5) for $T \rightarrow T_{\mathrm{c}}$.

In order to have nonzero solutions of Eq. (5) and the corresponding gap equation the effective coupling constant $\bar{g}=\mathcal{D}_{\bar{\mu}}\left(v^{2} / \xi_{0}-U\right)$ must be positive (here $\mathcal{D}_{\varepsilon}$ is the density of states (DOS) of the bare fermions, $\bar{\mu}=\mu-\frac{U}{2} n_{\mathrm{F}}$ ). Using square DOS one obtains, for $v \ll U, 2 D$, the critical value of $n$ below which superconducting solutions do not exist

$$
n_{\mathrm{c}}{ }^{U}=\frac{2 D+\Delta_{\mathrm{B}}-v^{2} / 4 U}{2 D+U} \text {. }
$$

On the other hand, the formal boundary between BCS and Bose regions obtained from a condition $\xi_{0}=\Delta_{\mathrm{B}}-2 \mu=0$ is given by

$$
n_{\mathrm{c}}=\frac{2 D+\Delta_{\mathrm{B}}}{2 D+U}
$$

(a reduction of $n_{c}$ with increase in $U$ is due to an upward Hartree shift, $\frac{U}{2} n_{\mathrm{F}}$, of the fermion band). From Eqs. (6) and (7) one concludes that for $U>0$ (and $v \ll U, 2 D)$ the BCS region is reduced to a small concentration range close to $n_{\mathrm{c}}$.

Influence of $U$ on $T_{\mathrm{c}}$ in the Bose region can be estimated for $v \ll U, 2 D$ and the limit of zero boson band width $\left(\xi_{k} \equiv \xi_{0}\right)$. At $T=T_{c}$ we have $n_{\mathrm{F}} \sim n_{\mathrm{c}}, n_{\mathrm{B}}=1 /\left[\exp \left(\xi_{0} / T_{\mathrm{c}}\right)-1\right] \sim \frac{1}{2}\left(n-n_{\mathrm{c}}\right)$. On the other hand, because $\vec{g} \ll 1, T_{\mathrm{c}}$ may be obtained (in both the BCS and Bose regions) from (5) as

*There is no need to include an on-site repulsion between bosons and fermions as they describe electron states which are well separated in space. 
$T_{\mathrm{c}}=1.14 \sqrt{D^{2}-\bar{\mu}^{2}} \exp (-1 / \bar{g})$. Using above equations to solve for $T_{\mathrm{c}}$ and $\xi_{0}$ one finally obtains

$$
T_{c}=\frac{\mathcal{D}_{\bar{\mu}} v^{2}}{\left(\bar{g}+\mathcal{D}_{\bar{\mu}} U\right) f(n)}, \quad \text { where } \quad f(n) \equiv \ln \left(1+\frac{2}{n-n_{\mathrm{F}}}\right)
$$

and where $\bar{g}$ is approximately given by

$$
\frac{1}{\bar{g}}=\ln \left[\frac{A}{\ln A}\left(\frac{1+\mathcal{D}_{\bar{\mu}} U \ln A}{1+\mathcal{D}_{\bar{\mu}} U}\right)\right], \quad A \equiv 1.14 \frac{\sqrt{D^{2}-\bar{\mu}^{2}}}{\mathcal{D}_{\bar{\mu}} v^{2}} f(n)\left(1+\mathcal{D}_{\bar{\mu}} U\right) .
$$

For very weak $v$ and $U \sim D, \bar{g}$ in (8) may be neglected and $T_{\mathrm{c}}$ is approximately given by a very simple formula: $T_{\mathrm{c}}=v^{2} / U f(n)$. Accuracy of the above general formulas ${ }^{\dagger}$ was verified for the weak $v$ using numerical results obtained with $\mathcal{D}_{\varepsilon}=\frac{3}{4 \sqrt{2}} \sqrt{\varepsilon+1}$ (corresponding to isotropic 3D free-particle-like spectrum: $\varepsilon_{q}^{0}=q^{2} / 2 m_{F}-D$ with a cut-off $q_{c},-1<\varepsilon<1, D \equiv 1$; see Fig. 1a). Despite the strong reduction of $T_{\mathrm{c}}$ with increase in $U$, superconductivity survives in this region at least as long as RPA theory is valid.

As concerns the nature of phase transition in the BF model one expects to find an observable contribution to the thermodynamic properties as $T \rightarrow T_{\mathbf{c}}$ coming from the condensing bosons. The RPA calculation of the boson Green function (Fig. 1b) shows that the bosons exhibit rather well defined quasiparticle features at $T_{\mathrm{c}}$. Decrease in the coupling $\bar{g}$ narrows the quasiparticle peak as it gets to small $\omega$ region. For a very small wave vector, $q<q_{0} \sim 2 q_{\mathrm{c}} T_{\mathrm{c}} / \mu$, the quasiparticle peak obeys $q^{2}$ dispersion law with relatively small value of an effective boson mass (see Fig. 1b, inset). In conclusion, we note that although the RPA method does not allow to study the region of the phase transition in a consecutive way due to a lack of its self-consistency, it seems to share some similarities with
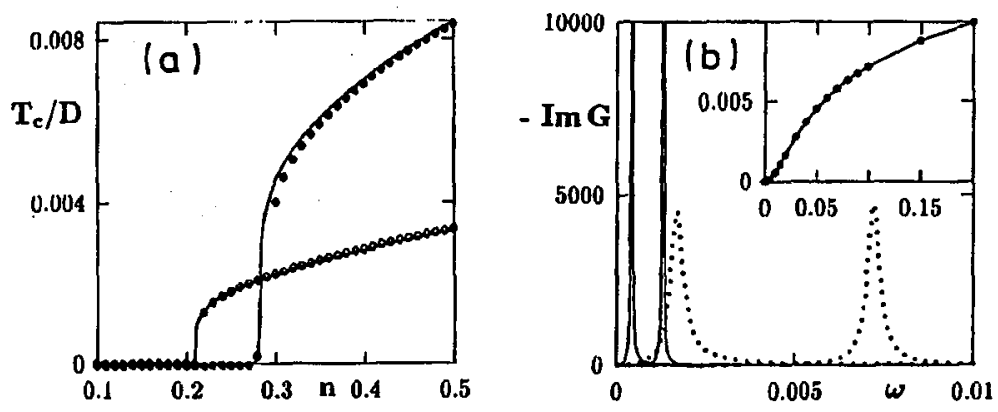

Fig. 1. (a) $T_{c}$ versus concentration $n$ for $v / D=0.1, \Delta_{\mathrm{B}}=-\frac{8}{9} D: \quad U=0(\bullet)$, $U=D(0)$. The symbols $(\bullet, 0)$ : approximate formulas $(8,9)$, lines: numerical solution of Eq. (5). (b) $-\operatorname{Im} G(q, \omega)$ for $v / D=0.1: U=0$ (dots), $U=D$ (lines) for $q / 2 q_{c}=0.02$, 0.1 . Inset: position of maximum of $-\operatorname{Im} G$ as a function of $q / 2 q_{\mathrm{c}}$ for $U=0(n=0.4$, $\left.T=T_{\mathbf{c}}\right)$.

tThe present approximation overestimates value of $T_{\mathrm{c}}$ in quasi-2D systems. Significant improvement in this respect would require a completely self-consistent approach [6]. 
a self-consistent calculations $[6,8]$ concerning the behavior of the bosons in the vicinity of condensation point.

\section{Acknowledgments}

I would like to thank R. Micnas, J. Ranninger and S. Robaszkiewicz for discussion. This work has financial support from the Committee for Scientific Research, project numbers 2 P03 B 05709 and 2 P03B 104 11. Thanks are also due to the Polish-French cooperation through KBN-French Embassy agreement.

\section{References}

[1] Y.J. Uemura, G.M. Luke, B.J. Sternlieb, J.H. Brewer, J.F. Carolan, W.N. Hardy, R. Kadono, J.R. Kempton, R.F. Kief, S.R. Kreitzman, P. Mulhorn, T.S. Riseman, D.L. Wiliams, B.X. Yang, S. Uchida, H. Takagi, J. Gopalakrishnan, A.W. Sleight, M.A. Subramanian, C.L. Chien, M.Z. Cieplak, Gang Xiao, V.Y. Lee, B.W. Statt, C.E. Stroniach, W.J. Kossler, X.H. Yu, Phys. Rev. Lett. 62, 2317 (1989).

[2] R. Micnas, J. Ranninger, S. Robaszkiewicz, Rev. Mod. Phys. 62, 113 (1990).

[3] T. Schneider, T. Keller, Phys. Rev. Lett. 69, 3374 (1992).

[4] J. Ranninger, S. Robaszkiewicz, Physica B 135, 468 (1985).

[5] S. Robaszkiewicz, R. Micnas, J. Ranninger, Phys. Rev. B 36, 180 (1987).

[6] J. Ranninger, J.M. Robin, M. Eschrig, Phys. Rev. Lett. 74, 4027 (1995).

[7] R. Friedberg, T.D. Lee, Phys. Rev. B 40, 423 (1989).

[8] J.M. Robin, Ph.D. Thesis, CNRS-CRTBT, Grenoble 1995, unpublished. 